\title{
Eine Krise in der Krise: Corona-Krisenkommunikation von Bürgermeister*innen in Deutschland
}

\author{
Stephan Habscheid • Friedemann Vogel
}

Angenommen: 8. Juni 2021 / Online publiziert: 2. August 2021

(C) Der/die Autor(en) 2021

Zusammenfassung Das politische System in Deutschland wird gegenwärtig durch eine zweifache Krise herausgefordert: Zum einen gilt es, die Bedrohung durch die Pandemie und deren gesellschaftliche Folgen durch praktische und kommunikative Maßnahmen zu bewältigen, wobei der Rechtsstaat und das Regierungshandeln in diversen Politikfeldern an ihre Grenzen kommen. Zum anderen stellen bereits seit geraumer Zeit politische Akteure, die für »das Volk« zu sprechen beanspruchen und als »Populisten « bezeichnet werden, in Frage, dass in der etablierten repräsentativen Demokratie eine ausreichende Repräsentation der Bürger bzw. eines völkisch gedachten Kollektivs gewährleistet sei. Von dieser zweifachen Krise, die in öffentlichen Corona-Protesten kulminiert, betroffen sind nicht zuletzt auch Bürgermeister*innen, die - im Idealfall - kommunikativ in verschiedenen Aufgabenfeldern des demokratischen Rechtsstaats agieren. Der Beitrag gibt erste Einblicke in ein Forschungsprojekt, das sich der Kommunikation von, mit und über Bürgermeister*innen systematisch widmet. Exemplarisch untersucht wird im vorliegenden Beitrag erstens, wie Bürgermeister*innen im Kontext der doppelten Krise in Sozialen Medien sprachlich agieren können und welche Anschlusskommunikation mit und unter Bürger*innen sich in den Kommentarlisten entwickelt. Zweitens wird danach gefragt, wie sich das abstrakte Bild der Bürgermeister*innen in der Presseberichterstattung gestaltet und im Zuge der Corona-Krise entwickelt. Zum Zweck des Vergleichs sowie im Hinblick auf Verflechtungen zwischen den beiden Gegenstandsbereichen werden Methoden der linguistischen Praxeologie, der interaktionalen Schriftlinguistik, der Analyse von Textkommunikation und der Korpus- und Imagelinguistik kombiniert.

\footnotetext{
Stephan Habscheid $(\square) \cdot$ Friedemann Vogel

Philosophische Fakultät, Germanistisches Seminar, Universität Siegen, Siegen, Deutschland

E-Mail: habscheid@germanistik.uni-siegen.de

Friedemann Vogel

E-Mail: friedemann.vogel@uni-siegen.de
} 
Schlüsselwörter Krisenkommunikation · Politolinguistik ·

Bürgermeisterkommunikation · Corona-Pandemie · Populismus · Soziale Medien ·

Praxeologie · Textkommunikation · Interaktionale Schriftlinguistik ·

Korpuslinguistik $\cdot$ Imagelinguistik

\section{A Crisis Within the Crisis: Corona Crisis Communication by Mayors in Germany}

Abstract The German political system is currently challenged by a twofold crisis: On the one hand, the threat caused by the pandemic and its social consequences have to be managed through practical and communicative measures, while the constitutional state and government action in various policy areas (health, education, economy, etc.) are approaching their limits. On the other hand, in recent years political actors who claim to advocate for »the people « and are referred to as »populists « have been questioning whether sufficient representation of citizens respectively a collective conceptualized in racist terms is guaranteed in established representative democracy. This twofold crisis, which culminates in the protests against governmental measures, also affects mayors, who - ideally - act communicatively in various fields of the democratic constitutional state. This article provides initial insights into a research project devoted to a systematic analysis of communication by, with and about mayors. First, the article examines how mayors can act linguistically in the context of the double crisis in social media and what kind of communication with and among citizens subsequently develops in the comment lists. Second, it asks how the abstract image of mayors in press coverage is shaped and developed in the course of the pandemic. For the purpose of comparison and with regard to interconnections between the two subject areas, methods of linguistic praxeology, analysis of written interaction and text communication as well as corpus and image linguistics are combined.

Keywords Crisis Communication · Political Linguistics · Mayoral Communication · Corona Pandemic $\cdot$ Populism - Social Media $\cdot$ Praxeology $\cdot$ Text Communication · Interactional Linguistics of Written Communication · Corpus Linguistics · Image Linguistics

\section{Eine zweifache Krise}

Das politische System der Bundesrepublik Deutschland ist aktuell in doppelter Hinsicht herausgefordert. Zum einen führen die Maßnahmen zur Bewältigung der Corona-Pandemie den Rechtsstaat, aber auch das Regierungshandeln in diversen Politikfeldern (Bildung, Wirtschaft etc.) immer wieder an ihre Grenzen (Krise 1), zum anderen stellen bereits seit einiger Zeit politische Akteure, die für »das Volk « zu sprechen beanspruchen, den Anspruch in Frage, dass in der etablierten repräsentativen Demokratie eine angemessene Repräsentation dieses (z.T. in völkischer Tradition gedachten) Kollektivs gewährleistet sei (vgl. Niehr/Reissen-Kosch 2018; Detering 2019) (Krise 2). In Protesten gegen staatliche Maßnahmen zur Eindäm- 
mung der Corona-Pandemie kulminieren beide Krisen, wenn etwa Vergleiche zu den Verhältnissen in einer »Diktatur « gezogen werden.

Mit der Krise, die auf den Begriff »Populismus« gebracht wird, befasst sich ein Forschungsprojekt im Sonderforschungsbereich $1472 »$ Transformationen des Populären« an der Universität Siegen (seit Januar 2021). ${ }^{1}$ Der Titel des Projekts, das von den beiden Autoren dieses Beitrags geleitet wird, lautet: »>Einer von uns < Diskursive Konstruktionen, Medien der Partizipation und sprachliche Praktiken der Bürgermeisterkommunikation in der Krise politischer Repräsentation «. ${ }^{2}$ Den analytischen Zugriff des SFBs aufgreifend, vermeiden wir eine normative und reifizierende Bestimmung von Populismus zugunsten eines erklärenden, rekonstruktiven und situationalen Ansatzes: Demnach fasst der Begriff Populismus situations- und perspektivenabhängig jeweils die »unerwünschte«, auch die als »bedrohlich« wahrgenommene Popularität von herausfordernden Positionen in der Perspektive etablierter Institutionen (vgl. Transformationen des Populären, S. 22-24). Derartige Phänomene gibt es nicht nur im Kontext der Politik, sondern z.B. auch in der Medizin oder in der Religion, weshalb im SFB im Plural von »Populismen« die Rede ist (ebd., S. $10)$.

Dass eine die Institutionen herausfordernde Popularität überhaupt erlangt werden kann, hängt mit Transformationen der Herstellung von Beachtung durch die Organisation von Öffentlichkeit zusammen, denen der SFB systematisch nachgeht. Für unser Thema relevant sind unter anderem die ökonomisch-technischen Mechanismen sozialer Medien (vgl. Gerlitz/Helmond 2013), die es strategisch agierenden und technisch-medial versierten Akteuren erlauben, Aufmerksamkeit in Form quantifizierter Bewertungen anderer zu erreichen und diesen Umstand, wiederum aufmerksamkeitsheischend, wirkungsvoll öffentlich in Szene zu setzen (vgl. Transformationen des Populären, S. 11, 14f.). In linguistischer Perspektive wird die bereits in der massenmedialen (politischen) Kommunikation zu beobachtende Tendenz fortgeführt, durch stilistische und sprachlich-ideologische Ressourcen Beachtung, Gefolg- und Gegnerschaft zu erzielen (vgl. etwa Klein 2014, S. 349-372), wobei normative Grenzen des Sagbaren, die in den Massenmedien durch einen berufsethisch gebundenen Journalismus gesichert werden (sollen), im Netz leichter zu überschreiten sind. Zu den sprachlichen und kommunikativen Phänomenen, die für Populismus als charakteristisch erachtet werden, gehören in diesem Zusammenhang etwa Simplifizierung (z.B. Verschwörungserzählungen), die Diffamierung politischer Eliten, Ausdruck und Anstachelung von Wut sowie - mit fließenden Übergängen zum (Rechts-)Extremismus - »Hate speech « und Gewalt-Phantasien, bis hin zu sprachlichen Straftaten (z.B. Beleidigung, Bedrohung), geschichtspolitische Provokationen, Antisemitismus und die Ausgrenzung von »Volksfeinden« und »Fremden« (vgl. detailliert Niehr/Reissen-Kosch 2018; Detering 2019). Hinzu kommen eine »Skandalisierung und Moralisierung « angeblicher »Nicht-Beachtung « der breiten Bevölkerung durch massenmediale »Gatekeeper« (Transformationen des Populären, S. 14). Im Ganzen wird so der Versuch unternommen, Populismus von seinen kommunikativen, me-

\footnotetext{
1 Gefördert durch die Deutsche Forschungsgemeinschaft (DFG) -SFB 1472 »Transformationen des Populären $\ll-438577023$.

2 Die zitierte Formulierung »Einer von uns« findet sich bei Gehne (2012, S. 133).
} 
dientechnisch-ökonomischen und sprachlichen Bedingungen her zu erklären, wobei die Mechanismen der Plattformen bzw. deren strategische Nutzung und der Sprachgebrauch ineinandergreifen.

Im Blick auf Reaktionen in den Institutionen, die sich durch den Populismus herausgefordert sehen, unterscheidet der SFB heuristisch zwischen $>$ Resilienz $<$, $>$ Resistenz $<$ und $>$ Akkomodation $<$ :

Resilienz heißt hier, das Populäre abprallen zu lassen (Endreß/Maurer 2015) und sich ostentativ unbeeindruckt zu zeigen; Resistenz bedeutet aktiven Einspruch gegen die Geländegewinne des Populären [...]. Akkomodation bezeichnet demgegenüber das Bemühen etablierter Ordnungen, sich den Ansprüchen des Populären anzupassen, es zu integrieren oder gar sich ihm anzuschmiegen.

In diesem konzeptuellen Rahmen gilt das Interesse unseres Projekts der Bürgermeisterkommunikation. Im öffentlichen Diskurs über Populismus kommen Bürgermeister*innen als eine Projektionsfläche der Diffamierung politischer Eliten, von populistischer Wut und von Hass ins Blickfeld, mitunter werden auch Kandidat*innen für das Bürgermeisteramt selbst als »Populisten« charakterisiert. Aufgrund ihrer relativen kommunikativen und sozialen Nähe zu den Bürger*innen (vgl. Weber 2017) und dem Alltagsbezug vieler kommunalpolitischer Themen wird kommunalpolitischen Mandatsträgern mehr als anderen professionellen Akteuren zugetraut, die populistische Herausforderung für das System abzufangen (vgl. Altenbockum 2016). Durch die Corona-Krise und ihre Verflechtung mit der schon länger bestehenden, vom Populismus angeheizten Krise der politischen Repräsentation haben es die Bürgermeister*innen nun mit einer noch größeren Herausforderung zu tun, können aber durch ihre spezifischen kommunikativen Ressourcen auch in besonderer Weise zu Bewältigung der Krise(n) beitragen. ${ }^{3}$

Vor diesem Hintergrund gibt der vorliegende Beitrag im Sinn einer Pilotstudie einen ersten Einblick in der Untersuchung zweier Gegenstandsbereiche, ihrer Verflechtungen und ihres Vergleichs: Zum einen gehen wir der Frage nach, wie Bürgermeister*innen im Kontext der zweifachen Krise sprachlich-kommunikativ agieren und mit den Bürger*innen interagieren, auch wie sich die kommentierende Anschlusskommunikation unter den Bürger*innen in Sozialen Medien gestaltet. Zum anderen fragen wir danach, ob, inwieweit und wie sich das Bild der Bürgermeister*innen in den Massenmedien während der Corona-Pandemie von der Zeit davor unterscheidet. In der sprachwissenschaftlichen Forschung zu Kommunikation in Institutionen bzw. Sprache in der Politik stellt die Untersuchung von Bürgermeisterkommunikation bislang noch weitestgehend ein Desiderat dar.

\footnotetext{
3 Vgl. zur Analyse eines Solidaritätsappells im Brief eines Bürgermeisters während der Coronakrise Liedtke 2020. Im Blick auf die Krisenkommunikation der Bürgermeister*innen zu berücksichtigen ist, dass im Fall kreiszugehöriger Städte in die kommunalpolitische Bewältigung der Pandemie auch die Landkreise eingebunden sind.
} 


\section{Methodenkombination}

Ausgangspunkt der Rekonstruktion von kommunikativem Sinn bildet das sozialtheoretische Prinzip der Praxis (vgl. Schüttpelz/Meyer 2017, 2018) bzw. Co-operative Action (Goodwin 2018): Beteiligte an der Herstellung von Sinn verfertigen wechselseitig Abläufe, indem sie jeweils die von ihren Vorgängern ins Spiel gebrachten, zeichenförmig geprägten materiellen Ressourcen (nicht zuletzt indexikalische Verweise auf komplexe kulturelle bzw. interaktionsgeschichtliche Verstehenshintergründe) partiell aufgreifen und in der Verfolgung ihrer Ziele transformierend wiederverwenden. Auf dieser Grundlage können sich mit dem Effekt einer Vereinfachung der Verständigung Konventionen herausbilden und verändern, Medien selbst - ohne die Kommunikation nicht denkbar ist - werden jedoch elementarer und dynamischer gefasst als »kooperativ erarbeitete Kooperationsbedingungen « (Schüttpelz 2016, S. 5). Derartige Prozesse liegen nach Goodwin (2018, S. 1) vielfältigsten Dimensionen und Aspekten des menschlichen (Zusammen-)Lebens zugrunde (vgl. auch Schüttpelz/Meyer 2018): Dazu zählen gleichsam kleinteilige Prozesse wie die multimodale und sequenzielle Interaktion, aber auch Sequenzen und Schichten von Handlungen und Diskursen im soziokulturellen Kontext bis hin zu Institutionen und der funktionalen Ausdifferenzierung von Gesellschaften. Goodwin zeigt auf, wie neue Handlungen systematisch ko-operativ hervorgebracht werden, also zustande kommen und verständlich werden durch sinnhaft strukturierende und geschichtete Operationen auf dem, was andere an Ressourcen und Lösungen für die Verständigung zuvor geschaffen haben (Goodwin 2018, S. 431; vgl. auch Schüttpelz/Meyer 2018, S. 179f.). Auf diese Weise können nach Goodwin Erträge menschlichen Handelns transformiert, akkumuliert und tradiert werden, und Akteurinnen können nur vor diesem Hintergrund ihre situative bzw. situationsübergreifende Handlungsmacht erlangen (Goodwin 2018, S. 440).

Wesentlich für das Konzept der Praxis und der Praktiken (Abläufe) ist die Verknüpfung von sprachlichem und praktischem Tun an der zeichenhaften Oberfläche mit historisch-kulturellen Verstehenshintergründen, wobei kommunikativer Sinn gleichermaßen reproduktiv wie stets dynamisch hergestellt wird (vgl. auch Habscheid 2016):

A doing or saying constitutes an X-ing [d.h. eine bestimmte sinnvolle Handlung; ISS] [...] against the background of an understanding of X-ing that is carried in some practice. The action, as a result, presupposes the practice concerned. lndeed, it is a moment of the practice. (Schatzki 2002, S. 96, mit dem erläuternden Zusatz zitiert nach Schulz-Schaeffer 2010, S. 323)

Methodisch ergibt sich hieraus, dass kommunikative Prozesse linguistischhermeneutisch ${ }^{4}$ rekonstruiert (aber auch im strengen wissenschaftlichen Sinn falsifiziert ${ }^{5}$ ) werden können anhand des in sie eingebetteten Gebrauchs von Zeichen, ihres situationalen Umfeldes, mitzubringender, ggf. auch ethnographisch zu erlangender relevanter Wissenshintergründe und der Positionierung der Zeichen

\footnotetext{
4 Vgl. zu einer Linguistischen Hermeneutik Hermanns 2012.

5 Vgl. zu diesem Anspruch einer rationalen Interpretation Eco 2004, S. 30.
} 
in den Abläufen und wechselseitigen Anschlussoperationen. Obwohl derartige Prozesse im idealtypischen Fall der Interaktion unter raumzeitlich Anwesenden an Körperlichkeit, soziomaterielle Umgebungen und umfassende wechselseitige Wahrnehmungswahrnehmungen gebunden sind, lassen sich auch technisch vermittelte semiotische Prozesse wie die sprach-, wahrnehmungs- und wissensabhängige »Textkommunikation « (vgl. Hausendorf et al. 2017), die durch Erweiterungen und Begrenzungen der kommunikativen Potentiale gekennzeichnet sind, als Ausprägungen ko-operativer Praxis verstehen und rekonstruieren.

Hierbei ist die jeweilige technische Medialität noch differenzierter zu berücksichtigen. So greift die kommunalpolitische Kommunikation von Bürgermeister*innen zunehmend auf Plattformen im Internet zurück. Zu diesen Plattformen zählen sowohl unidirektionale Informationsangebote (kommunale Websites, parteipolitischgerahmte Personenprofilseiten oder Informationsservice-Apps) als auch stärker auf Dialog und Teilhabe ausgerichtete Angebote in Sozialen Medien (Facebook-Profilseiten, Bürger-Chats, Twitter-Accounts u.ä.) oder eigens entwickelte (freie oder kommerzialisierte) Partizipationsplattformen. Die vorliegende Untersuchung konzentriert sich in ihrem praxeologischen Teil auf die Kommunikation eines Bürgermeisters bei Facebook und knüpft an die medienlinguistische bzw. interdisziplinäre CMC-Forschung an, wie sie sich spätestens seit den 1990er Jahren etabliert hat (Runkehl/Schlobinski/Siever 1998; Herring/Stein/Virtanen 2013; Schmitz 2015). Webbasierte Kommunikationsumgebungen sind dabei (potentiell) multimodal, heteroglossisch und intertextuell organisiert (Androutsopoulos 2010) und bewegen sich - je nach Kommunikationsform und Textsorte/Gattung - auf einem breiten Spektrum zwischen prototypischer Interaktion und Textkommunikation. Zur adäquaten Rekonstruktion dieser sprachlich-kommunikativen Aktivitäten ist es notwendig, verschiedene Datentypen miteinander ins Verhältnis zu setzen, nämlich (mindestens) 1) die Einbettung der sprachlichen Äußerungen in einen multimodalen Text einschließlich der schriftlich-visuellen Sehflächen und 2) die teilweise interaktiv aufeinander bezogenen sprachlichen Äußerungen in den Kommentarlisten. ${ }^{6}$ Für die vorliegende Pilotstudie wird die Kommunikation des Oberbürgermeisters der Stadt Dresden im März 2020, in der Anfangsphase der Corona-Pandemie, herangezogen. Im Blick auf die Anonymisierung der Beiträge der privaten Plattformnutzer*innen werden internetlinguistische Zitierkonventionen aufgegriffen (vgl. Marx/Weidacher 2014, S. $18-23)$.

Komplementär zur praxeologischen, qualitativen Untersuchung von Bürgermeisterkommunikation im Kontext der Corona-Krise wird in einer korpus- bzw. imagelinguistischen Analyse (vgl. Vogel 2017) nach dem abstrakten, öffentlichen Bild von Bürgermeister*innen und seiner Veränderung im Zusammenhang mit der CoronaPandemie gefragt. Grundlage hierfür sind in der vorliegenden Pilotstudie massenkommunikative Texte aus 17 überregionalen und regionalen Zeitungen und Zeitschriften aus zwei Zeiträumen, die über die Mediendatenbank LexisNexis und auf

\footnotetext{
6 In der Hauptstudie sollen darüber hinaus durch die Kombination mit Expertenbefragungen Daten aus für Plattformnutzer*innen nicht einsehbaren administrativen Backend-Oberflächen und -aktivitäten (z.B. Sperrungs- oder Löschungspraktiken) berücksichtigt werden.
} 
Tab. 1 Primärkorpus der korpus- und imagelinguistischen Teilstudie

\begin{tabular}{ll}
\hline Primärkorpus & Textmenge \\
Medium & 4969 \\
\hline Rheinische Post & 2009 \\
Kölner Stadt-Anzeiger & 721 \\
Stuttgarter Zeitung & 681 \\
Stuttgarter Nachrichten & 500 \\
taz, die tageszeitung & 425 \\
Südwest Presse & 424 \\
Spiegel Online & 401 \\
Berliner Zeitung & 266 \\
B. Z. & 229 \\
Die Welt & 116 \\
BILD.de & 110 \\
Die ZEIT (inklusive ZEIT Magazin) & 91 \\
Der Spiegel & 71 \\
Die Welt am Sonntag & 53 \\
Sächsische Zeitung & \\
Stammausgabe Dresden & 25 \\
Focus Magazin & 15 \\
BILD am Sonntag &
\end{tabular}

Basis des Suchausdrucks *Bürgermeister* semiautomatisch erhoben und aufbereitet wurden (vgl. Tabelle 1).

Auf diese Weise entstand ein Primärkorpus aus insg. 11.106 Texten aus dem Zeitraum vom 01.03. bis 31.07.2020 (»Pandemie-Korpus«), außerdem ein sekundäres Vergleichskorpus mit insgesamt 4.233 Texten aus dem vorpandemischen Zeitraum vom 01.03. bis 31.07.2019. Die unterschiedliche Korpusgröße resultiert daraus, dass für beide Teilkorpora zwar der identische zeitliche Querschnitt, aber im Vergleichskorpus nicht jeweils der gesamte Monat, sondern aus arbeitsökonomischen Gründen immer nur Texte eines 10-Tageintervalls (10.-19.03.2019; 10.-19.04.2019 usw.) erhoben wurden. Darüber hinaus gibt es in beiden Korpora sehr große Unterschiede in der Verteilung der Texte nach Medium, die sich nur bedingt etwa aus fehlendem Regional- oder Kommunalbezug erklären lassen. Im Primärkorpus etwa macht der Textumfang allein von Rheinischer Post und Kölner Stadt-Anzeiger rund $63 \%$ des Gesamtkorpus aus. Die wenigsten Belege finden sich in Wochenzeitungen bzw. -magazinen (Focus, Spiegel, Zeit, Wochenend-Blätter), aber zum Beispiel auch in der Sächsischen Zeitung (mit Schwerpunkt Dresden). In einer derzeit in Vorbereitung befindlichen Hauptstudie wird dieser Unwucht weiter nachgegangen. Alle Texte von Primär- und Vergleichskorpus wurden mithilfe des TreeTagger (Schmid 1994) POSannotiert und - je nach Teilinteresse - mit den korpuslinguistischen Analysetools CorpusExplorer, AntConc (Anthony 2012) und LDA-Tookit (Vogel 2012) ausgewertet.

Neben den selbsterhobenen Textkorpora wurden komplementär das Deutsche Referenzkorpus (DeReKo) und das cOWIDplus-Korpus des IDS Mannheim, das Diskursmonitor-Live-Korpus, das Zeitungskorpus des DWDS sowie Google Ngrams für 
eine indizienbasierte Bildung, Erhärtung oder Widerlegung von Hypothesen herangezogen.

Auf Basis der verschiedenen Korpora wurden Keywords, Key-Ngramme und kontrastive Kookkurrenzen zur Ausgangszeichenkette [Bb]ürgermeister (einschließlich aller Komposita) berechnet und anschließend durch ergänzende Volltextsichtungen interpretativ eingeordnet. Durch die gewählten Tools und Daten-kontrastierenden Algorithmen konnten auf diese Weise jene Ausdrücke ermittelt werden, die zur Pandemiezeit im statistischen Sinne überzufällig häufig gemeinsam mit Bürgermeister*innen gebraucht wurden im Unterschied zu Texten aus dem VorpandemieZeitraum - und umgekehrt. Da dieses kontrastive Verfahren zugleich jene Ausdrücke bzw. Kotextmuster aus dem Blickfeld verdrängt, die in beiden Zeiträumen im Verhältnis zur jeweiligen Datengrundgesamtheit gleichermaßen belegt sind, wurden auch nicht-kontrastive Kookkurrenzanalysen zu beiden Korpora durchgeführt.

\section{Linguistische Praxeologie: Bürgermeisterkommunikation in sozialen Medien}

Im etablierten politischen System kommen Bürgermeister*innen im Wesentlichen drei Rollen zu, die mit entsprechenden kommunikativen Aufgabenfeldern und hierzu benötigten sprachlichen und weiteren semiotischen Ressourcen einhergehen:

a) Bürgermeister*innen sind zunächst die Leiter der kommunalen Verwaltung und deren öffentliche >Galionsfiguren`(Gehne 2012). Sie können sich mehr als jeder andere kommunale Akteur die in der Verwaltung gebündelten Fachkompetenzen und rechtlich-administrativen Problemlösungsressourcen auf ihre Fahnen schreiben, müssen aber auch für charakteristische systemische Verständigungsprobleme, Zumutungen und Grenzen der Inklusion (Burzan et al. 2008) in der Bürger*innenkommunikation »den Kopf hinhalten «. Die hierauf beruhenden Konflikte können sich unter den Bedingungen der zweifachen Krise kommunikativ beträchtlich verschärfen, müssen aber auch unter dem konkreten Entscheidungsund Handlungsdruck auf der kommunalen Ebene im Modus einer pragmatischen Verwaltung (vgl. Seibel 2016) situativ bewältigt werden.

b) Als Führungspersönlichkeiten (und Moderatoren) im Stadtrat und in der lokalen Öffentlichkeit setzen Bürgermeister*innen deutliche kommunalpolitische, mitunter auch parteipolitische Akzente (Gehne 2012). Während im Wahlkampf bis zur Wahl ein kompetitiver, mehr oder weniger zugespitzter Modus der Abgrenzung kommunikativ funktional sein kann, ist der/die Bürgermeister*in im Amt im Interesse der politischen Problemlösung auf vielfältige Kooperationen mit (ausgewählten) politischen Konkurrenten, institutionellen und privaten Akteuren angewiesen.

c) Schließlich kommen, weit mehr als die politischen Akteure in der europäischen, der Bundes- oder der Landespolitik, Bürgermeister*innen - »vor allem in kleineren und mittleren Städten und Gemeinden, die immer noch das Bild von Kommunalpolitik in vielen Regionen Deutschlands prägen « (Gehne 2012, S. 52) - auf einer interpersonalen Ebene auch »menschlich« (Weber 2017) mit Bürger*innen 
in Kontakt. Teilweise in Verbindung mit traditionellen Ritualen wie Gedenkreden, Festreden oder Grußworten (vgl. Antos 1986, 1987), massenmedialer Inszenierung und der Interaktion in sozialen Medien knüpfen Bürgermeister*innen an populäre Freizeitbeschäftigungen an (Radtouren, Spaziergänge, Sportveranstaltungen, Stadtfeste mit Pop-Elementen etc.) und etablieren im Erfolgsfall das Image, nicht nur »einer für uns «, sondern auch »einer von uns« zu sein (Gehne 2012). Auf diese Weise können systemische Probleme der Teilhabe durch affektive Kommunikationsweisen (vgl. Werber 2016) symbolisch bearbeitet werden. Gleichzeitig wird durch die Inszenierung des Kollektivs aber auch ein symbolischer Ankerpunkt für populistische Kritik und Abgrenzung etabliert.

Die im Rahmen der praxeologischen Pilotstudie rekonstruierten kommunikativen Praktiken sind diesen drei Aufgabenfeldern zuzuordnen.

\subsection{Aufgabenfeld 1: Kommunale Verwaltung}

Wie in einem Beispiel aus einem Politik-Lehrbuch tritt in Beispiel 1 der Bürgermeister als Vermittler zwischen den Bürger*innen, der Verwaltung und der Politik sowie zwischen der kommunalen Ebene und der - mit dieser im Bereich der Exekutive verfassungsrechtlich verflochtenen - Landesebene in Erscheinung. Auf seiner Facebook-Seite, die sich - so die Begründung - für eine öffentliche »virtuelle Bürgersprechstunde« nicht nur aufgrund ihrer Reichweite (»biete ich allen Dresdnerinnen und Dresdnern [...] an«), sondern auch angesichts der Corona-bedingten Einschränkungen (»es gilt ja Abstand zu halten«) in der Situation doppelt gut eignet, unterbreitet er das Angebot, für die komplexen Maßnahmen auf Nachfrage öffentlich Rede und Antwort zu stehen (vgl. Abb. 1). Adressiert werden dabei nicht nur

Abb. 1 Initiierung der Bürgersprechstunde (FB_Hilbert_ 05_20200324)

\begin{abstract}
(6) Dirk Hilbert
24. März 2020 Q

Zu den umfangreichen Regeln und Einschränkungen haben uns in den letzten Tagen eine große Zahl an Rückmeldungen erreicht. Viele befürworten die Maßnahmen, aber es gibt auch Unsicherheit und Fragen. Deshalb biete ich allen Dresdnerinnen und Dresdnern eine virtuelle \#Bürgersprechstunde an (es gilt ja Abstand zu halten): Senden Sie mir einfach bei Facebook Ihre \#Fragen und ich werde in einem Video hier bei Facebook antworten.
\end{abstract}

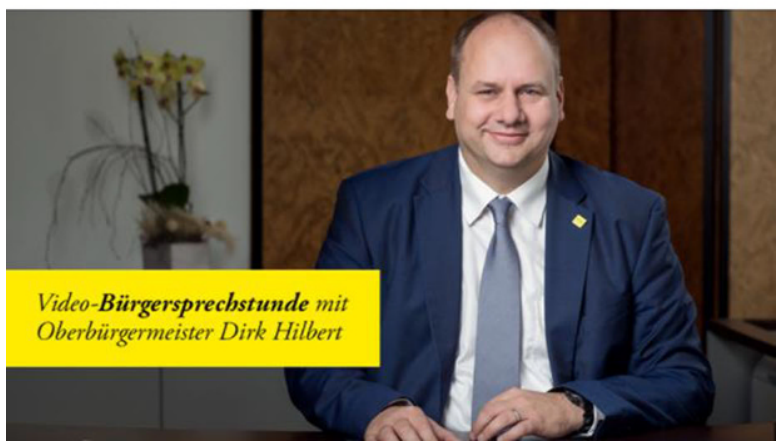


Abb. 2 Interaktion in der virtuellen Bürgersprechstunde (FB_Hilbert_05_20200324)
D079

Sehr geehrter Herr Hilbert, warum wurden nun auch noch - und nur in Dresden- die Wochenmärkte geschlossen, wo man im Freien mit dem entsprechenden Abstand

frisches Obst, Gemüse und Fleisch aus der Region kaufen kann. ?

( ich gehe immer nur auf den Ma... Mehr ansehen Gefällt mir - Antworten - 18 Wochen

Dirk Hilbert

D079 Liebe FrauD079, die Schließung der Wochenmärkte war nicht mein Wunsch und Wille. Ich halte die Schließung auch nicht für sinnvoll. Vielmehr ist es eine Anordnung des Freistaates Sachsen, die wir Folge zu leisten haben. Herzliche Grüße, Dirk Hilbert

Gefällt mir - Antworten - 18 Wochen

\section{D079}

Dirk Hilbert

Vielen Dank für Ihre schnelle Antwort.

An wen kann ich mich nun direkt wenden?... Mehr ansehen

Gefällt mir - Antworten - 18 Wochen

Dirk Hilbert

D079 Staatsministerien für Soziales und Inneres.

Gefällt mir - Antworten - 18 Wochen

Antworten ...

die Befürworter, sondern auch Bürger*innen, die unsicher sind und Fragen haben dezidierte Kritiker werden in dieser Inszenierung des städtischen Kollektivs dagegen nicht explizit genannt.

An das im Videoformat vermittelte Angebot sequenziell anschließend, fordert eine Bürgerin (D079) in Form einer Warum-Frage in Verbindung mit durch wo - im Sinn von obwohl - eingeleiteten Gegenargumenten Rechenschaft ein (vgl. Abb. 2).

Der Bürgermeister schließt sich ihrer Kritik explizit an (»Ich halte die Schließung [der Wochenmärkte] auch nicht für sinnvoll.«). Er verzichtet auf den Versuch einer Legitimation, sondern grenzt sich stattdessen mit einer bekräftigenden Paarformel (»nicht mein Wunsch und Wille«) nachdrücklich von der kritisierten Entscheidung ab. Er verweist auf die Verantwortung der Landesregierung für diese Entscheidung und auf den sachlichen Umstand, dass die kommunale Verwaltung in diesem Fall bloß Ausführende landesrechtlich verbindlicher Vorgaben ist.

Die Bürgerin würdigt in ihrem Folgezug die rasche und responsive Auskunft und bekräftigt durch die Nachfrage »An wen kann ich mich nun direkt wenden? «ihr Ansinnen einer Partizipation an der konkreten Verwaltungsentscheidung. Als Antwort auf ihre Frage erhält sie vom Bürgermeister die präzisierende Auskunft, welche Instanzen in der Landesregierung für die zur Rede stehende Anordnung verantwortlich sind (»Staatsministerien für Soziales und Inneres«). Dass kein weiterer sequenzieller Zug der Bürgerin mehr folgt (auch keine Kommentare von dritter Seite), kann als 
Indiz dafür gewertet werden, dass der Bürgermeister dem Anliegen der Bürgerin an dieser Stelle entsprochen hat.

Ohne etwas über die mögliche Fortsetzung der Kommunikation auf der Landesebene und ihr Ergebnis zu wissen, lässt sich allgemein sagen, dass derartige Praktiken den Bürger*innen die Erfahrung ermöglichen können, in Entscheidungsprozesse als interaktive Phänomene eingebunden zu sein und so intensiver als nur durch periodische, abstrakte Wahlentscheidungen an öffentlicher Verwaltung bzw. Politik zu partizipieren (vgl. Hausendorf/Bora 2006; zur konversationellen MikroOrganisation von Partizipation u.a. Mondada/Svensson/van Schepen 2015). Im Kontext der zweifachen Krise kann dem eine doppelte Relevanz zukommen.

\subsection{Aufgabenfeld 2: Kommunale Politik}

Wie Bürgermeister kommunalpolitische, teilweise auch parteipolitische Akzente setzen, zeigt Beispiel 2, wiederum von der Facebook-Seite des Dresdner Oberbürgermeisters. Der Kommunal- und FDP-Politiker berichtet über und argumentiert für seine Initiative im Stadtrat (»[...] Deshalb schlage ich dem Stadtrat vor ...«), die auf eine »Unterstützung von Kleinstunternehmen, Selbständigen und Freiberuflern « in der Corona-Krise zielt. Dass die Hilfe »kurzfristig und unbürokratisch« erfolgen soll, verweist indexikalisch und musterhaft auf den Wissenshintergrund der besonderen Handlungsanforderungen in einer Krise (vgl. Abb. 3).

Wie im Bereich der Verwaltung schließt sich auch auf der Ebene der kommunalen Politik ein interaktiver Klärungs- und Aushandlungsprozess an (vgl. Abb. 4).

Die Interaktion ist zum Teil gekennzeichnet durch ausgeprägte Höflichkeit: Nachfragen statt Forderungen (»Haben Sie bereits eine Vorstellung ...? ), Ausdruck von Verständnis für sachlich begründete Verzögerungen, Dank und gute Wünsche (mit einem Glücksklee-Emoji) in der existenziellen Krisensituation (Äußerungen von D004). Einen vorwurfsvollen Ton mit emotionalisierender Interpunktion, aber ohne

Abb. 3 Ankündigung einer politischen Initiative zur Krisenbewältigung (FB_Hilbert_01_20200319)

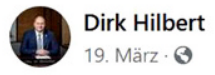

Das Coronavirus stellt uns als Stadtgesellschaft vor ungeahnte Herausforderungen. Die Dresdnerinnen und Dresdner müssen wissen, dass die Stadt für sie da ist. Deshalb schlage ich dem Stadtrat vor, eine Soforthilfe zur Unterstützung von Kleinstunternehmen,

Selbstständigen und Freiberuflern auf den Weg zu bringen. Gerade sie sind besonders davon betroffen, dass wir das öffentliche Leben so stark einschränken müssen. Einnahmen und Aufträge brechen komplett weg, aber die laufenden Kosten bleiben. Hier wollen wir als Stadt die Betroffenen mit je 1.000 Euro kurzfristig und unbürokratisch helfen.

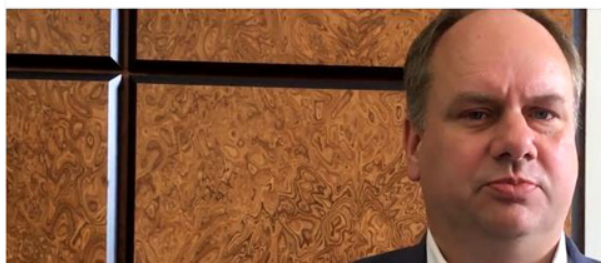


Abb. 4 Ein politischer Klärungs- und Aushandlungsprozess (FB_Hilbert_01_20200319)

\section{D004}

Dirk Hilbert haben Sie bereits eine Vorstellung bis wann Sie ein KleinSTunternehmen werten? Analog dem Paragraph 19 Absatz 2 UStG?

Gefällt mir · Antworten · 19 Wochen

(1) Autor/in

An den Details der Richtlinie arbeiten gerade die

Fachleute - bitte haben Sie ein wenig Geduld,

Stadtrat ist kommenden Donnerstag.

Gefällt mir · Antworten · 19 Wochen

D004

Dirk natürlich. Vielen Dank für Ihre Antwort und bleiben Sie gesund. 옹

Gefällt mir · Antworten · 19 Wochen

D014

Die Kleinunternehmer wurden vergessen!

Gefällt mir · Antworten · 18 Wochen

D014

Also die nebenberuflich tätigen, die allesamt Steuern zahlen!

populistische Eskalation (Wut, Partner-Abwertung etc.), schlagen die letzten beiden der zitierten Äußerungen (von D014) an.

\subsection{Aufgabenfeld 3: Repräsentation der städtischen >Gemeinschaft «}

Ein traditionelles Verfahren von Bürgermeister*innen, um lokale Popularität gleichzeitig zu erlangen und zugleich auf Akteure und Themen zu verteilen, besteht im Vollzug von Anerkennungsritualen, zu denen beispielsweise die im Alltag (noch immer) weit verbreiteten Grußworte gehören. Dabei handelt es sich um ein »ein mehrfach rückgekoppeltes System von Geben und Nehmen« (Antos 1987, S. 19): Die Rituale setzen Anlass und Veranstaltung voraus und bewirken (deklarativ) deren offizielle und öffentliche Wertschätzung, die sie (expressiv) zum Ausdruck bringen. Zugleich verdeutlichen sie die Funktion des Amtsträgers und bieten ihm durch gewisse Gestaltungsspielräume die Möglichkeit, seine Politik »bevölkerungsnah « in Szene zu setzen (Antos 1986; Antos 1987). Die ethnomethodologisch inspirierten, textlinguistischen Studien aus den 1980er beschreiben wesentliche Merkmale der Gattungsfamilie, lassen aber im Blick auf die Gegenwart die Frage offen, ob und inwieweit die traditionellen Verfahren der Relevanzzuschreibung durch populistische Diskurse (z.B. gegen »abgehobene« politische Eliten) und durch neuartige Formate der Popularisierung von Akteuren und Wissen (z.B. in sozialen Medien) unter Druck geraten.

Beispiel 3 zeigt den Versuch des Bürgermeisters, unter Corona-Distanzbedingungen Zusammenhalt stiftende Rituale in den Kontext sozialer Medien zu übertragen und die Rituale dabei gleichsam zu demokratisieren, indem nicht nur Amtsträger in Form performativer Sprechakte, sondern jede Bürgerin und jeder Bürger öffentlich, 
Abb. 5 Hamsterkauf (FB_ Hilbert_03_20200323)

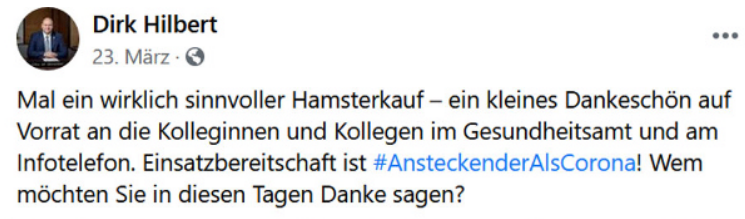

D047
Ich möchte meiner Familienhilfe Danke sagen. Sie ist
weiter im Einsatz... und bringt uns morgen Stoffbänder
und Molton mit.
Gefältt mir · Antworten · 19 Wochen · Bearbeitet
D048
Ganz klar dem Busfahrer der mich täglich zur Arbeit
bringt.
Gefällt mir · Antworten · 19 Wochen
D049
Das dürfte dem ganzen nicht gerecht werden aber ist ein
Anfang. Weiter so 0
Gefällt mir · Antworten · 19 Wochen
D050
Das haben sich die Kollegen auch mehr als verdient ...
Gefällt mir · Antworten · 19 Wochen

auf der Facebook-Seite des Bürgermeisters anderen Bürger*innen Dank zollen und sie auf diese Weise ehren können. In einem Diskurskontext zu Beginn der Pandemie, in dem viel von problematischen »Hamsterkäufen« (z.B. von Toilettenpapier) die Rede war, leitet der Bürgermeister zur Gewinnung von Aufmerksamkeit ein öffentliches Dankesritual für städtische Mitarbeiter*innen mit einem scherzhaften Verweis auf den Hamsterkauf-Diskurs ein (»Mal ein wirklich sinnvoller Hamsterkauf $[. ..] \ll)($ vgl. Abb. 5).

Einer auf die Pandemie-Krise bezogenen, spezifischen Kontextualisierung des Dankesrituals dient nicht nur die Auswahl der Geehrten (»Gesundheitsamt«, »Infotelefon«), sondern auch der Hashtag-Bezug auf die Kampagne der Stadt für Zusammenhalt in der Krise, »\#Ansteckender als Corona«. Der Post schließt mit der Aufforderung an die Bürger*innen, ihrerseits - gleichsam mit offiziellem Rückenwind des Bürgermeisters - öffentliche Dankesrituale für im Krisenkontext engagierte Mitbürger*innen zu realisieren. Auch die medienabhängige Möglichkeit, den Dank anderer durch Likes öffentlich zu bekräftigen, von der hier einige Beteiligte Gebrauch machen, trägt zu dieser Demokratisierung der öffentlichen Rituale, die Zusammenhalt stiften sollen, bei.

Während in der Anschlusskommunikation einerseits viele Bürger*innen das Dankesritual des Bürgermeisters begrüßen (vgl. in Abb. 5 z.B. die Äußerungen von D049 und D050) und/oder es durch eigene öffentliche Dankesbekundungen an Personen in ihren persönlichen Netzwerken fortsetzen (vgl. z.B. die Äußerungen von D047 und D048), äußern andere mittels indexikalischer Verweise die Kritik, dass es sich beim Dank an das städtische Personal um eine nur symbolische und zwischenmenschliche 
Abb. 6 Kleine Geschenke (FB_ Hilbert_03_20200323)

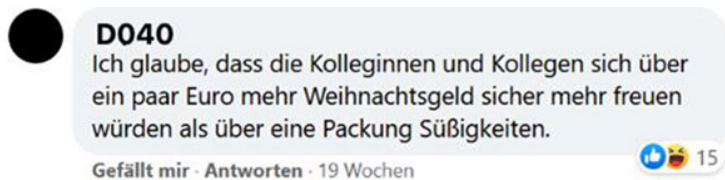

Gefällt mir - Antworten - 19 Wochen

\section{D041}

D040 Das Weihnachtsgeld bzw. die Jahressonderzahlung ist tariflich vorgegeben und kann nicht einfach willkürlich angepasst werden. Schließlich werden die MitarbeiterInnen von Steuergeldern finanziert. Des Weiteren glaube ich, dass jede Form der Aufmerksamkeit und Anerkennung begrüßenswert ist, auch wenn es sich dabei nicht um Geld handelt.

Gefällt mir · Antworten · 19 Wochen

D D 42

D040 kleine Geschenke erhalten die Freundschaft :-

Gefällt mir - Antworten - 19 Wochen

Geste (»eine Packung Süßigkeiten«), nicht um eine organisationale Maßnahme (vgl. Habscheid et al. 2015) im Umgang mit dem städtischen Personal (»Weihnachtsgeld «) handelt (vgl. Abb. 6). Die Äußerung stößt ihrerseits auf Widerspruch, wobei der von Vereinfachung (einem Merkmal des Populismus) geprägte Charakter der Kritik ebenso zurückgewiesen wird wie die Abspaltung des Persönlichen vom Organisationalen (vgl. die Bedeutung der verwendeten Redensart »Kleine Geschenke erhalten die Freundschaft« in der Äußerung von D042).

Die diskursbezogene Grenze zum (Rechts-)Populismus wird deutlich überschritten in Äußerungen, die - thematisch z.T. nur locker an die rituelle Aktion des Bürgermeisters anknüpfend - hintergründige Bezüge zum stereotyp verkürzenden Diskurs über Einwanderer als Sozialstaatsbelastung und Sicherheitsrisiko bzw. zu Verschwörungstheorien über die Ursache der Pandemie (»Biowaffenangriff «) herstellen (vgl. Abb. 7).

Ganz ohne expliziten Bezug zu den konkreten Anlässen und Themen der Bürgermeisterkommunikation bleiben Äußerungen, die den repräsentativen Status des Bürgermeisters als solchen zu delegitimieren versuchen (»Nicht mein Bürgermeister «) und/oder den Bürgermeister in derber Weise diffamieren (»Was'n das fuer ein Stotterfritze «). Interessant ist dann zu sehen, wie solche Äußerungen teilweise von anderen User*innen zurückgewiesen werden, woran sich Streitsequenzen innerhalb der Kommentare anschließen können. Diese verweisen unter Umständen nicht nur auf die Populismus-Krise, sondern auch auf die besonderen Umstände der Corona-Krise, wobei Letztere nicht nur als Verschärfung der ersteren, sondern auch als deren Relativierung perspektiviert wird. In der Kommentarliste zu einem Post des Bürgermeisters über gesteigerte Maßnahmen zur Eindämmung der Corona-Krise in der Landeshauptstadt findet sich die folgende Interaktionssequenz (vgl. Abb. 8).

D032 wertet hier den Bürgermeister durch die delegitimierende Formel nach dem »Nicht mein «-Muster der Protestkommunikation in Verbindung mit einem FäkalienEmoji in derber Weise ab. Daran schließt sich eine mit humoristischer Überlegenheit 
Abb. 7 Laden Deutschland (FB_Hilbert_03_20200323)

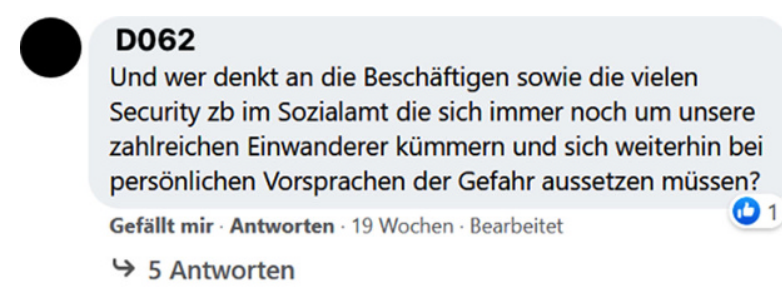

Abb. 8 Sitzen bleiben!

(FB_Hilbert_02_20200320)

\section{D063}

Man muss allen danken die den Laden Deutschland am laufen halten, denn wenn wir diesen Biowaffenangriff überstehen sollten, brauchen wir jede Hand.

Gefällt mir · Antworten · 19 Wochen

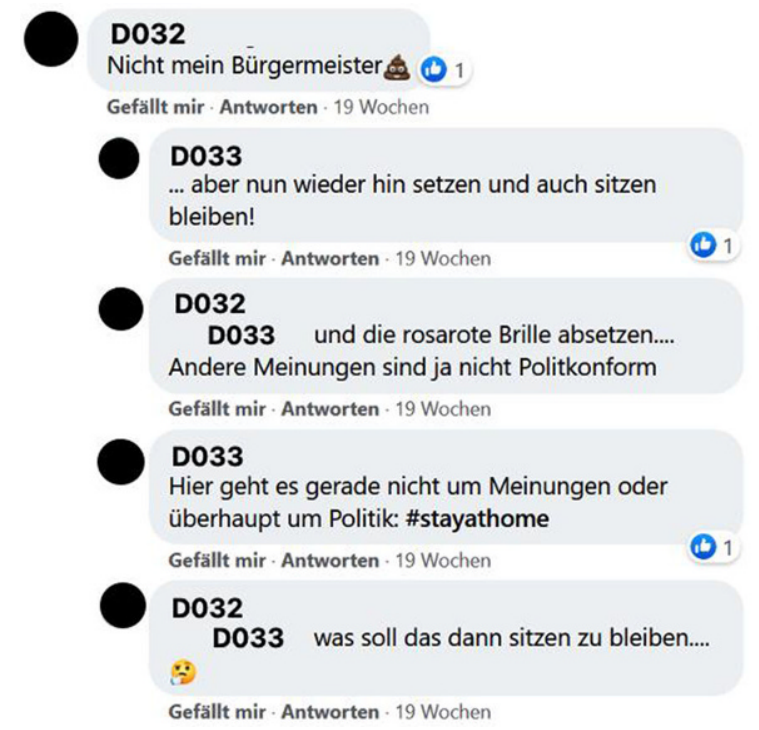

auf den Schulkontext verweisende Aufforderung von D033 zum »setzen und auch sitzen bleiben« an. Der Betroffene reagiert humorlos und formelhaft mit explizitem Verweis auf den Politik-Kontext und wird darauf hin durch D032 erneut belehrt: Im größeren Kontext der Pandemie und ihrer Bewältigung (»\#stayathome«) in einer frühen Phase werden »Meinungen « und »überhaupt Politik « als deplatziert charakterisiert. Die Corona-Krise wird hier herangezogen, nicht um die Populismus-Krise zu verschärfen, sondern um sie gleichsam rhetorisch auszubremsen.

\section{Korpus- und Imagelinguistik: Das Bild der Bürgermeister*innen in der Presse}

Fragen wir nun - zum Vergleich mit den kommunikativen Praktiken - nach dem sprachlich-kommunikativen Bild von Bürgermeister*innen in der Corona-Pandemie, 
wie es sich imagelinguistisch in massenkommunikativen Texten nachzeichnen lässt. Korpus- und imagelinguistisch stehen dabei die beiden folgenden erkenntnisleitenden Fragen im Vordergrund:

1. $\mathrm{Zu}$ Krise 1: Inwiefern unterscheiden sich rekurrente Sprach(gebrauchs)muster im kotextuellen Umfeld von Bürgermeister*innen während der Pandemie von jenen Sprachgebrauchsmustern im Zeitraum vor Beginn der Pandemie? Welche Eigenschaften werden Bürgermeister*innen in der Pandemie im Kontrast zum Vorpandemiezeitraum zugeschrieben, welche kommunalen Praktiken im massenkommunikativ vermittelten Bild hervorgehoben? Methodisch gewendet: Welche Coronakrisen-bezogene und Krisen-unabhängige Keywords und Key-Ngramme lassen sich im Umfeld von Bürgermeister*innen erheben und als ausdrucksseitige Indikatoren für ein pandemiespezifisches Image von Bürgermeister*innen und ihren kommunalen Verwaltungen interpretieren?

2. Zu Krise 2: Finden sich quantitative Indizien für die Auseinandersetzung von Bürgermeister*innen mit populistischen Aktivitäten, wenn ja, in welchem Umfang?

Die korpuslinguistischen Analysen zeigen: Die Präsenz von Bürgermeister*innen in öffentlichen Medien folgt offenbar erwartbaren wie auch (einstweilen noch) nichtvorhersehbaren Aufmerksamkeitsperioden, die besonders durch bestimmte Ereignisse oder Themen getriggert werden. Einen ersten Eindruck dazu vermitteln diachrone Frequenzverläufe des Ausdrucks *Bürgermeister* in den verschiedenen externen Datensammlungen (DWDS, Google Ngrams, cOWID, Diskursmonitor): Interessanterweise scheinen Bürgermeister*innen in den späten 1990er und frühen 2000er Jahren vorübergehend besondere Aufmerksamkeit zu erhalten, zumindest in den Zeitungskorpora des DWDS (vgl. Abb. 9). Die kurzfristigeren Datenbestände aus

\section{*Bürgermeister* - Verlaufskurve}

Basis: DWDS-Zeitungskorpus

150

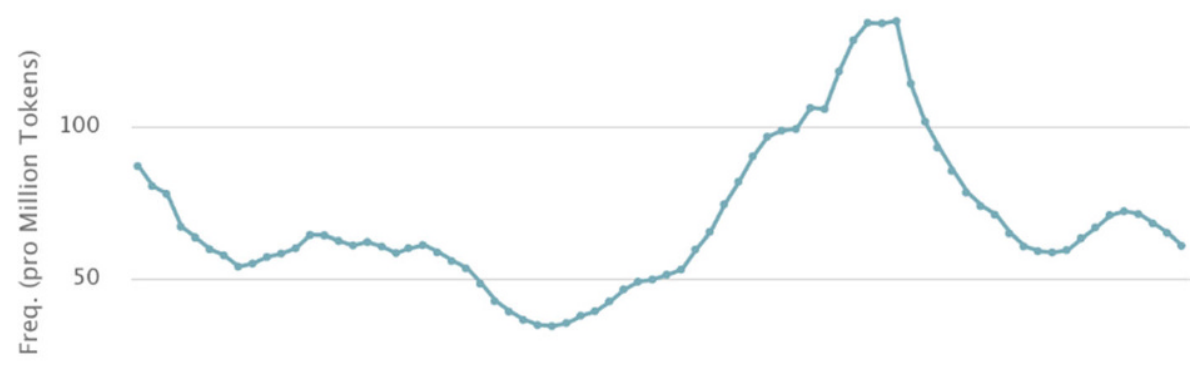

0

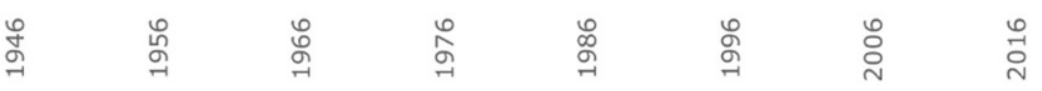

$\rightarrow$ *Bürgermeister": Zeitung

Abb. 9 Langfristige Verlaufskurve 


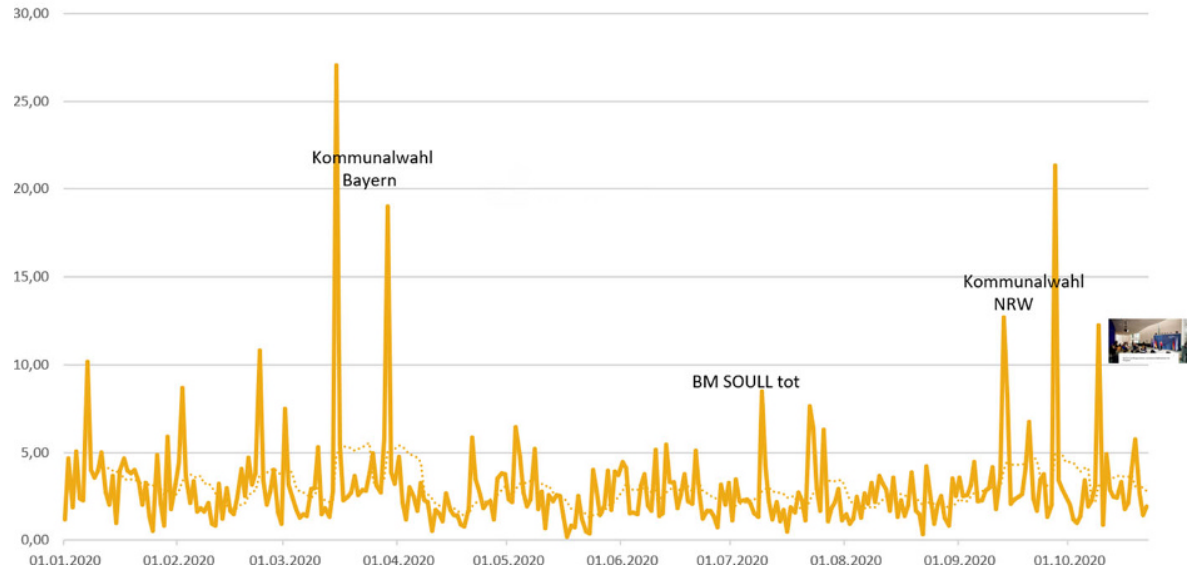

Abb. 10 Kurzfristige Verlaufskurve

dem Pandemiezeitraum (Diskursmonitor, cOWIDplus) weisen darauf hin, dass es zum Beispiel Kommunalwahlen, aber auch >Schicksalsschläge< oder Themen mit landes- oder bundesweiter Bedeutung sind, die den Fokus auf Bürgermeister*innen lenken (vgl. Abb. 10). In der Pandemie korreliert die Frequenz der Nennung von Bürgermeister*innen zeitweise auch mit der Häufigkeit von Pandemie-bezogenen Schlagwörtern (Maßnahme, Lockdown) (vgl. Abb. 11), was auf einen medial konstituierten Zusammenhang zwischen Pandemie-Entwicklung/Bekämpfung und der Aufgaben/Rollen von Bürgermeister*innen hinweisen könnte.

Mit ziemlicher Sicherheit wären sowohl mit Blick auf Intervalllänge als auch hinsichtlich Themen und Anlässen Unterschiede nach Medienreichweite zu konstatieren, das wird allein schon an der Ungleichgewichtung der Korpusgrößen von überregionalen und regionalen Zeitungen ersichtlich. Hier sind differenziertere Un-

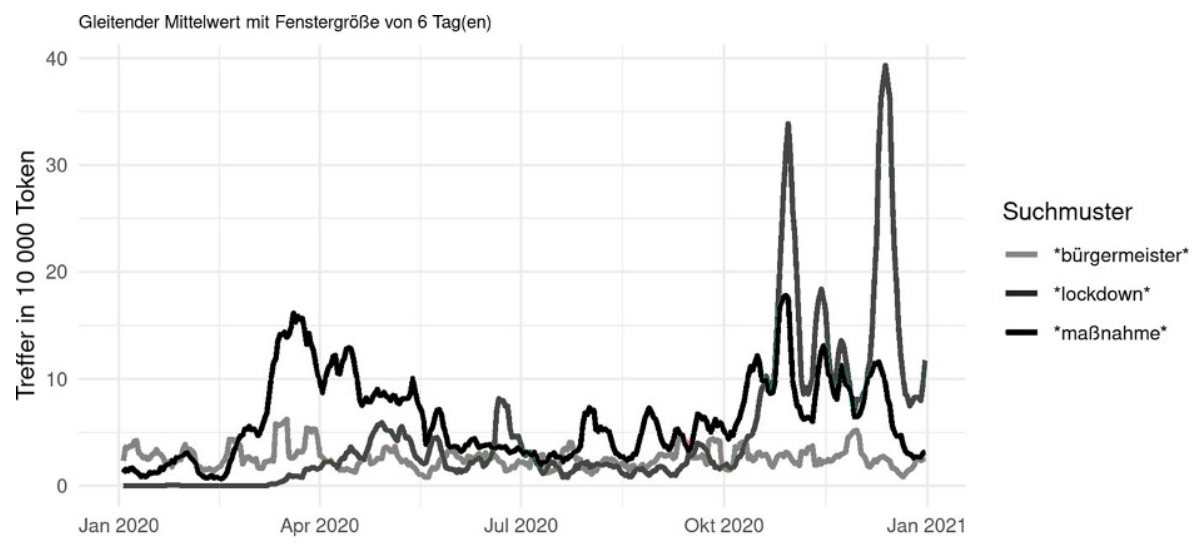

Abb. 11 Frequenz-Korrelationen 
Tab. 2 Wortformen und Komposita

\begin{tabular}{llll}
\hline Ausdruck & $\mathrm{f}$ & Ausdruck & $\mathrm{f}$ \\
\hline Bürgermeister & 11077 & Ortsbürgermeister & 41 \\
Bürgermeisterin & 1673 & Baubürgermeister & 41 \\
Bürgermeisterkandidat & 1084 & Baubürgermeisterin & 32 \\
Oberbürgermeister & 524 & Ordnungsbürgermeister & 29 \\
Bürgermeisteramt & 289 & Bezirksbürgermeisterin & 24 \\
Bürgermeisterwahl & 271 & Bürgermeisterkandidatur & 23 \\
Bürgermeisterkandidatin & 209 & Finanzbürgermeister & 19 \\
Bezirksbürgermeister & 98 & Bürgermeisterbüro & 16 \\
Oberbürgermeisterin & 47 & Oberbürgermeisterwahl & 15 \\
Bürgermeisterposten & 42 & Bürgermeisterschaft & 14 \\
\hline
\end{tabular}

tersuchungen nötig (etwa auch zur Verknüpfung von singulären kommunalen Ereignissen zu transsituativen, also überregional wirksamen Narrativen).

Die 20 meistgebrauchten Wortformen und Komposita zu [Bb]ürgermeister im Primärkorpus verweisen auf unterschiedliche hierarchische Positionen von Bürgermeisterrollen und Zuständigkeitsbereiche, typische Orte und Ereignisse mit wiederkehrendem medialem Interesse (Tabelle 2).

Einen Überblick zu wiederkehrenden medial reproduzierten Ko(n)texten von Bürgermeister*innen gewinnt man durch die Erhebung und grobe semantische Kategorisierung von signifikanten Kookkurrenzen (also überzufällig im sprachlichen Umfeld verwendeten Wörtern) zu Bürgermeister-Nennungen im Primärkorpus (Personennamen im Einzelnen werden im Folgenden nicht berücksichtigt):

- generell zahlreiche Namen und Temporalia (Amtszeit): langjährig, amtierender, ehemaliger, scheidend usw.

- Akteure (Personen, Organisationen): derjenig, Infizierte, Stadtmusikant, Wachmann, Tochterunternehmen, Innenministerin, Kanzleramtschef, Lieferdienst, Lanz, Sterbende, Gesundheitsministerin, Wutbürger, Virologen, Besatzungsmitglied, Robert-Koch-Institut, Länderchef, Schwiegereltern

- Kognitive Haltungen: unvernünftig, standhaft, besonnen, Nachlässigkeit, Umsicht, hoffnungsvoll, tröstlich, trügerisch, Augenmaß, schonungslos, Eigenverantwortung, Führungsstärke

- Orte: Ratssitzung, Kreisrat, Finanzausschuss usw.; mancherorts, Plaza, Friseursalon, Tirol, Skigebiet, div. -straßen, Gesundheitsverwaltung, Ostküste, Stadion, Bergamo, Lebensmittelgeschäft, Labore, Grenzland

- Aktivitäten / Prozesse / Handlungen: betonen, überreichen, wählen, danken, bedanken, erklären, loben, begrüßen, wenden, kandidieren, erläutern, unterzeichnen, anschreiben, bitten, ernennen, gratulieren, kündigen, angegriffen, überreicht, verständigen, würdigen, ankündigen, verkünden, gerichtet, überrascht, informiert, wegschauen, wiederwählen, aussprechen, nachziehen, unterlassen, wegschicken, Grußwort, Schichtbetrieb, Nennung, Stationierung, Pressegespräch, Videobotschaften, Entschuldigung, zusammenstehen, unentdeckt, einschränkend, Arztbesuch, Publikumsverkehr, Zusammenführung, angezeigt, delegieren, Vorlie- 
gen, Gruppenbildung, Eheschließung, verordnet, hamstern, grasen, zuschalten, Erlass, Nachtschicht, Gassi, Trainingsbetrieb, aufweichen, durchregieren, vorpreschen, Anleihe, Simulation, Sofortmaßnahme, Soforthilfe, genesen, separieren, herunterspielen u.a.

- Objekte: Brandbrief, Urkunde, Bürgertelefon, Todesanzeige, Photos, Videobotschaften, Toilettenpapier, Strandkorb, Telefon-, Mangelware, Atemmaske, Schutzmaske

- Ereignisse: Dringlichkeitssitzung, Messerattacke, Trauergottesdienst, Verdachtsfall, Krisenfall, Ausnahmefall, Krisensituation, Turbulenz, AfD-Veranstaltung, Dringlichkeitsbeschluss, Lieferengpass

- Vorschriften / Normen: Meldepflicht, Verordnung, Erlass, Dringlichkeitsbeschluss, Ausgehverbot, Dekret

- Weitere Abstrakta: Lagerkapazität, Belastungsgrenze, Staatsangehörigkeit, Bewegungsfreiheit, Legitimität, Kolonialismus, Lichtblick, Wertigkeit, Wirksamkeit u.a.

- Graduierungen: schätzungsweise, triftig, martialisch, exponentiell, gefahrlos

Zahlreiche Kookkurrenzen zeigen bereits die Verknüpfung von BürgermeisterKontexten mit Sachverhalten der Pandemie, nicht nur regionalen, sondern auch überregionalen (Medien-)Ereignissen, Personen und Aktivitäten. Allerdings bleibt durch das so gewählte Analyseverfahren zunächst offen, welche Ko(n)texte besonders typisch für den Pandemie- und zugleich untypisch für die Vor-Coronazeit sind. Dies erlaubt eine kontrastive Kookkurrenzanalyse, bei der diejenigen Ausdrücke statistisch sichtbar werden, die relativ zur Korpusgröße besonders häufig im pandemischen Primärkorpus im Vergleich zur (relativen) Häufigkeit im Vergleichskorpus belegt sind. Die Ergebnisse deuten zumindest partiell auf einen grundlegenden Wandel in der medialen Perspektive auf Bürgermeister*innen-Aktivitäten hin: Standen im Vorpandemie-Zeitraum vor allem >Inklusion< und >Partizipation<-akzentuierende Aktivitäten im Vordergrund, sind es im Pandemie-Zeitraum besonders >(Aus)Schließungen $<$; das >Danken $<$ und >Grüßen $<$ weicht in der Corona-Zeit ein Stück weit dem >Auffordern $<$, >Ermahnen $<$ und $>$ Verbieten $<($ vgl. Tabelle 3$)$.

Bürgermeister*innen werden in der Pandemie-Zeit offenbar perspektiviert als verantwortliche Handlungsträger, die - für ihre sonstige Rolle - unübliche und tendenziell Ablehnung antizipierende Vorschriften erlassen, durchsetzen und legitimieren müssen. Zwar spielen bisherige ritualisierte Praktiken weiter eine Rolle (siehe die nicht-kontrastierenden Kookkurrenzen oben), aber sie stehen in der medialen Wiedergabe in ernsthafter Konkurrenz mit auf > Verwaltung < und > verneinende Verfügung r reduzierte Praktiken, die den tradierten Rollenerwartungen an Bürgermeister*innen widersprechen. Hierzu gehört auch, was auf den ersten Blick als mittlerweile weit verbreitete Kompensationsversuche eingeordnet werden könnte: der Ausgleich von wegfallender räumlich-physischer Präsenzkommunikation durch mediengestützte Kommunikationsformen. Auch Bürgermeister*innen halten im Bild der Presse nunmehr Telefon- und Videokonferenzen, nutzen WhatsApp und soziale Medien zur Bürger*innen-Kommunikation. Das geschieht mitunter aber nicht reibungslos, wie der folgende Beleg aus der Leonberger Kreiszeitung vom 07.06.2020 illustriert, zumal im Kontext von Auseinandersetzungen: 
Tab. 3 Kookurrenzen

\begin{tabular}{|c|c|}
\hline $\begin{array}{l}\text { Kontrastive Kookkurrenzen im } \\
\text { Vergleichskorpus (Vor-Pandemie- } \\
\text { Zeitraum) }{ }^{\mathrm{a}}\end{array}$ & $\begin{array}{l}\text { Kontrastive Kookkurrenzen im Primärkorpus (Pandemie-Zeit- } \\
\text { raum) }\end{array}$ \\
\hline $\begin{array}{l}\text { - Aktivitätsverben: eröffnen, emp- } \\
\text { fangen } \\
\text { - Nomen: Eröffnung, z.B.: Brun- } \\
\text { nensession, Ausstellung, Ge- } \\
\text { werbemarkt, Debatte usw.; Be- } \\
\text { grüßung / Grußwort: v.a. in } \\
\text { Veranstaltungsberichten; Fass- } \\
\text { anstich; Sprechtag; Erinnerung, } \\
\text { Schirmherr u.a. }\end{array}$ & $\begin{array}{l}\text { - Aktivitätsverben: absagen; erklären, berichten, mitteilen, } \\
\text { appellieren, empfehlen, fordern, entscheiden, erlassen, gelten, } \\
\text { verfügen, einhalten, unterstützen, bedauern } \\
\text { - Nomen: Appell, Schließung [von Veranst./Einricht.], [einheitl.] } \\
\text { Vorgehen, Erlass, Videokonferenz, Telefonkonferenz } \\
\text { - Adjektive und Nomen kognitiver Eigenschaften mit erziehe- } \\
\text { rischer/moralisierender Funktion: unvernünftig, standhaft, } \\
\text { besonnen, Nachlässigkeit, Umsicht, hoffnungsvoll, tröstlich, } \\
\text { trügerisch, Augenmaß, schonungslos, Eigenverantwortung, } \\
\text { Führungsstärke } \\
\text { - Partikeln: nicht X = stattfinden, unterstützen, eröffnen usw.; } \\
\text { X wegen/aufgrund } \mathrm{Y} \text { mit X = außerübliche Aktivität und } \mathrm{Y}= \\
\text { Corona, Maßnahme, Raumenge usw.; per X = außerordent- } \\
\text { liche Kommunikationsbedingungen (Telefon, Video, Brief, } \\
\text { WhatsApp) }\end{array}$ \\
\hline
\end{tabular}

${ }^{a}$ Die Anzahl signifikanter kontrastiver Kookkurrenzen ist für den Vor-Pandemie-Zeitraum erwartungsgemäß sehr viel kleiner analog zum deutlich kleineren Korpusumfang (siehe Methodik) im Vergleich zum Primärkorpus; die Analyse ist daher nur begrenzt aussagekräftig für das mediale Bürgermeister*innen-Bild außerhalb der Pandemie.

Gleichwohl musste Leonberg als Genehmigungsbehörde den Protestierern [Querdenken-Demo] eine adäquate Alternativfläche anbieten. Ein zu kleiner Platz, etwa das Reiterstadion, wäre angesichts der angemeldeten Dimensionen zu klein gewesen. So ist die Rechtslage. Deshalb ging Oberbürgermeister Martin Georg Cohn (SPD) mit dem Golfplatz ins Rennen. Hier ist genug Platz, um auch bei Wahrung des obligatorischen Abstands von 1,50 Metern maximal 7500 Menschen zuzulassen. Eine Entscheidung, die dem OB viel verbale Prügel einbrachte. Etliche Anwohner liefen gegen die Standort-Auswahl Sturm, diverse Stadträte reagierten pikiert, weil Cohn die Fraktionsspitzen nur per WhatsApp informiert hatte.

Tab. 4 Wortfeld um populistisch

\begin{tabular}{llllll}
\hline Ausdruck & $\mathrm{Chi}^{2}$ & $\begin{array}{l}\mathrm{f}(\text { PreCoro- } \\
\mathrm{na})\end{array}$ & $\begin{array}{l}\mathrm{f}(\text { Coro- } \\
\mathrm{na})\end{array}$ & $\begin{array}{l}\mathrm{f}(\text { PreCoro- } \\
\mathrm{na}) / 10.000\end{array}$ & $\begin{array}{l}\mathrm{f}(\text { Coro- } \\
\mathrm{na}) / 10.000\end{array}$ \\
\hline rechtspopulistisch & 2,596 & 2 & 0 & 219,780 & 0,000 \\
populistische & 2,007 & 6 & 3 & 659,341 & 256,410 \\
Populisten & 1,882 & 27 & 25 & 2967,033 & 2136,752 \\
Populistenmanöver & 1,292 & 1 & 0 & 109,890 & 0,000 \\
populistischem & 1,292 & 1 & 0 & 109,890 & 0,000 \\
populistischer & 0,650 & 2 & 1 & 219,780 & 85,470 \\
rechtspopulistischer & 0,550 & 3 & 2 & 329,670 & 170,940 \\
Rechtspopulisten & 0,110 & 22 & 26 & 2417,582 & 2222,222 \\
Rechtspopulist & 0,098 & 3 & 3 & 329,670 & 256,410 \\
\hline
\end{tabular}


Welche Mittel- oder Langzeitkonsequenzen die veränderte mediale Perspektive für die Wahrnehmung und kommunalen Konfliktfelder von Bürgermeister*innen haben wird, lässt sich anhand der hier herangezogenen Datenstichprobe nicht ablesen.

Zur Frage nach medial konstituierten Konfrontationen zwischen Bürgermeister*innen und populistischen Aktivitäten scheint sich das Bild teilweise zu verändern. Das substantivische und adjektivische Wortfeld rund um populistisch ist sowohl vor als auch in der Pandemie Bestandteil der Berichterstattung, zumindest in den hier zur Verfügung stehenden Pilotkorpora (vgl. Tabelle $4^{7}$ ).

Gleichwohl scheint das Thema Populismus von anderen (mutmaßlich Pandemiebezogenen) Themen ein Stück weit verdrängt zu werden (das passte auch zur in der Pandemie beobachtbaren Abnahme der medialen Präsenz von AfD und vergleichbaren Gruppen ${ }^{8}$ ). Möglicherweise hat sich aber auch nur das Wortfeld selbst verschoben hin zu Ausdrücken und Stigmawörtern wie Querdenker, Verschwörungstheoretiker, Aluhut u.ä. Dies wäre im Rahmen einer qualitativen Auswertung dieser und weiterer Daten zu prüfen.

\section{Ein (Zwischen-)Fazit}

Die praxeologische Auswertung der Facebook-Daten zeigt ein breites Spektrum von kommunikativen Aktivitäten des Bürgermeisters, die im Rahmen der klassischen Aufgabenfelder der Bürgermeisterkommunikation - Stadtverwaltung, Kommunalpolitik, Repräsentation der Stadtgemeinschaft - angesiedelt sind und darauf zielen, zur gleichzeitigen Bewältigung beider Krisen in ihrer Verwobenheit beizutragen. Erstens positioniert sich der Bürgermeister im Blick auf die Ausgestaltung der Corona-Maßnahmen als ein Vermittler zwischen den Bürger*innen, der Verwaltung und der Politik sowie zwischen der kommunalen und der mit dieser rechtlich verflochtenen Ebene der Landesregierung. Er ermöglicht den Bürger*innen die Erfahrung, in Verwaltungsentscheidungen als interaktive Prozesse eingebunden zu sein (vgl. Hausendorf/Bora 2006), was im Blick auf beide Krisen zur Erlangung von Legitimation beitragen kann. Zweitens setzt der Bürgermeister auch im Kontext der Bewältigung der Pandemie sowohl kommunalpolitische als auch parteipolitische Akzente. Wie bei der Beteiligung an Verwaltungsentscheidungen schließen sich auch auf kommunalpolitischer Ebene interaktive Klärungs- und Aushandlungsprozesse an, wie auch durch Populismus befeuerter, unsachlicher Streit. Drittens nutzt der Bürgermeister die Kommunikation bei Facebook, um - in Verbindung mit einer Kampagne für Zusammenhalt - unter Corona-Distanzbedingungen integrative Rituale, die an die offizielle Rolle des Bürgermeisters/der Bürgermeisterin gebunden sind, in den Kontext sozialer Medien zu übertragen; diese werden zugleich in gewisser Weise demokratisiert, indem nicht allein der offizielle Amtsträger, sondern jede Bürgerin

\footnotetext{
7 Die Spalte $2\left(» \mathrm{Chi}^{2} \ll\right)$ gibt den $\mathrm{Chi}^{2}$-Signifikanzwert an; die Spalten 3 und 4 geben die absolute, die Spalten 5 und 6 die relative Häufigkeit (pro 10.000 Token) der jeweiligen Ausdrücke an.

${ }^{8}$ So auch die Wahrnehmung bei Medienpraktikern, vgl. etwa tagesschau.de vom 23.03.2020 (https://www. tagesschau.de/faktenfinder/corona-populisten-101.html, 23.04.2021).
} 
und jeder Bürger auf der Facebook-Seite des Bürgermeisters eingeladen werden, anderen Bürger*innen Dank zu zollen und sie auf diese Weise öffentlich zu ehren.

In der Interaktion in den Kommentarlisten greifen viele User*innen die kommunikativen Angebote des Bürgermeisters auf den drei Aufgabenfeldern konstruktiv auf und führen diese jeweils sequenziell fort. Es finden sich aber auch Beiträge, die den repräsentativen Status des Bürgermeisters als solchen zu delegitimieren versuchen und/oder den Bürgermeister in derber Weise diffamieren. Aufschlussreich ist dann zu sehen, wie solche Äußerungen teilweise von anderen User*innen als populistische Stör-Aktionen zurückgewiesen werden, woran sich Streitsequenzen innerhalb der Kommentare anschließen können. Hier wird die Corona-Krise einerseits herangezogen, um die Populismus-Krise zu verschärfen, aber auch, um sie als situativ unangemessen aus dem Raum des Sagbaren auszuschließen.

Die korpuslinguistischen Imageanalysen deuten dazu passend auf die Rolle der Bürgermeister*innen als wichtigen Protagonisten der Pandemie-Bewältigung hin. Allerdings scheint das Bild in den Massenmedien insofern etwas verkürzt zu sein, als im Vergleich zum Vorpandemie-Zeitraum weniger >Inklusion< und >Partizipation $<-$ akzentuierende Aktivitäten wie >Eröffnen $<$, >Empfangen $<$, >Danken $<$ und >Grüßen $<$ im Vordergrund stehen, sondern eher die potenziell unpopulären, wie deren Negation, >(Aus)Schließungen $<$, >Absagen $<$, > Verfügungen $<$ oder ermahnende $>$ Appelle $<$. Von den zentralen Aufgabenfeldern der Bürgermeisterkommunikation wird offenbar stärker die hoheitliche Verwaltung, weniger die soziale Integration betont.

Die populistische Herausforderung scheint, geht man von ihrer expliziten Erwähnung aus, im öffentlichen Diskurs von anderen (die Pandemie betreffenden) Themen bis zu einem gewissen Grad verdrängt zu werden, lebt aber unter Umständen in Form anderer Wortfelder weiter. Dafür spricht auch ihre Fortführung und Thematisierung auf der praxeologischen Ebene der Facebook-Kommunikation, wo sie sich u.a. in Simplifizierungen (Weihnachtsgeld statt Schokolade), verschwörungstheoretischen Erklärungen der Pandemie (»Biowaffenangriff«), formelhaften Kampagnen gegen die kommunale Elite (»Nicht mein Bürgermeister«), stereotype Fremdenfeindlichkeit sowie persönlichen Abwertungen und Diffamierungen des Amtsträgers manifestiert, aber auch in klaren Zurückweisungen durch andere Bürger*innen.

Funding Open Access funding enabled and organized by Projekt DEAL.

Open Access Dieser Artikel wird unter der Creative Commons Namensnennung 4.0 International Lizenz veröffentlicht, welche die Nutzung, Vervielfältigung, Bearbeitung, Verbreitung und Wiedergabe in jeglichem Medium und Format erlaubt, sofern Sie den/die ursprünglichen Autor(en) und die Quelle ordnungsgemäß nennen, einen Link zur Creative Commons Lizenz beifügen und angeben, ob Änderungen vorgenommen wurden.

Die in diesem Artikel enthaltenen Bilder und sonstiges Drittmaterial unterliegen ebenfalls der genannten Creative Commons Lizenz, sofern sich aus der Abbildungslegende nichts anderes ergibt. Sofern das betreffende Material nicht unter der genannten Creative Commons Lizenz steht und die betreffende Handlung nicht nach gesetzlichen Vorschriften erlaubt ist, ist für die oben aufgeführten Weiterverwendungen des Materials die Einwilligung des jeweiligen Rechteinhabers einzuholen.

Weitere Details zur Lizenz entnehmen Sie bitte der Lizenzinformation auf http://creativecommons.org/ licenses/by/4.0/deed.de. 


\section{Literatur}

Altenbockum, Jasper von (2016): Heimat - Heilmittel gegen Globalisierung? In: FAZ.NET, 05.12.2016. URL: http://www.faz.net/aktuell/politik/harte-bretter/harte-bretter-ueber-globalisierung-fluchtpunktkommune-14558644.html, letzter Zugriff am 23.05.2020.

Androutsopoulos, Jannis (2010): Multimodal - intertextuell - heteroglossisch: Sprach-Gestalten in »Web 2.0«-Umgebungen. In: Arnulf Deppermann/Angelika Linke (Hg.): Sprache intermedial. Stimme und Schrift, Bild und Ton. Berlin: de Gruyter, S. 419-446.

Anthony, Laurence (2012): AntConc (3.2.4w) [Computer Software]. Waseda University. Tokyo. URL: http://www.antlab.sci.waseda.ac.jp/, letzter Zugriff am 22.05.2013.

Antos, Gerd (1986): Zur Stilistik von Grußworten. In: Zeitschrift für Germanistische Linguistik 14, S. $50-81$.

Antos, Gerd (1987): Grußworte in Festschriften als »institutionale Rituale«. In: Wolfgang Klein (Hg.): Sprache und Ritual. Zeitschrift für Literaturwissenschaft und Linguistik (LiLi) 65, S. 9-40.

Burzan, Nicole/Lökenhoff, Brigitta/Schimank, Uwe/Schöneck, Nadine M. (2008): Das Publikum der Gesellschaft. Inklusionsverhältnisse und Inklusionsprofile in Deutschland. Wiesbaden: Verlag für Sozialwissenschaften.

Detering, Heinrich $\left({ }^{4} 2019\right)$ : Was heißt hier »wir«? Zur Rhetorik der parlamentarischen Rechten. Stuttgart: Reclam.

Eco, Umberto ( $\left.{ }^{2} 2004\right)$ : Zwischen Autor und Text. Interpretation und Überinterpretation. München: dtv (Original: Interpretation and Overinterpretation, 1992).

Endreß, Martin/Andrea Maurer (Hg.) (2015): Resilienz im Sozialen. Theoretische und empirische Analysen. Wiesbaden: Springer.

Gehne, David H. (2012): Bürgermeister. Führungskraft zwischen Bürgerschaft, Rat und Verwaltung. Stuttgart: Boorberg.

Gerlitz, Carolin/Helmond, Anne (2013): The Like economy: Social buttons and the data-intensive web. In: New Media \& Society 15 (8), S. 1348-1365.

Goodwin, Charles (2018): Co-Operative Action. New York: Cambridge University Press.

Habscheid, Stephan (2016): Handeln in Praxis. Hinter- und Untergründe situierter sprachli-cher Bedeutungskonstitution. In: Arnulf Deppermann/Helmuth Feilke/Angelika Linke (Hg.): Sprachliche und kommunikative Praktiken. Berlin/New York: De Gryuter, S. 127-151.

Habscheid, Stephan/Müller, Andreas P./Thörle, Britta/Wilton, Antje (2015): Sprache in Organisationen. In: Ekkehard Felder/Andreas Gardt (Hg.): Handbuch Sprache und Wissen. Berlin/Boston: de Gruyter (Handbücher Sprachwissen, 1), S. 392-410.

Hausendorf, Heiko/Bora Alfons (Hg.) (2006): Analysing citizenship talk. Social positioning in political and legal decision-making processes. Amsterdam/Philadelphia: Benjamins.

Hausendorf, Heiko/Kesselheim, Wolfgang/Kato, Hiloko/Breitholz, Martina (2017): Textkommunikation: ein textlinguistischer Neuansatz zur Theorie und Empirie der Kommunikation mit und durch Schrift (RGL, 308). Berlin: de Gruyter.

Hermanns, Fritz (2012): Linguistische Hermeneutik. Überlegungen zur überfälligen Einrichtung eines in der Linguistik bislang fehlenden Teilfaches. In: Hermanns, Fritz: Der Sitz der Sprache im Leben: Beiträge zu einer kulturanalytischen Linguistik. Hg. von Heidrun Kämper/Angelika Linke/Martin Wengeler. Berlin/Boston: de Gruyter, S. 67-102.

Herring, Susan C./Dieter Stein/Tuija Virtanen (Hg.) (2013): Pragmatics of computer-mediated communication. Berlin: de Gruyter (= Handbook of pragmatics, 9).

Klein, Josef (2014): Grundlagen der Politolinguistik. Ausgewählte Aufsätze. Berlin: Frank \& Timme.

Liedke, Frank (2020): Wirus oder: Was es heißt, solidarisch zu sein. In: Themenheft: Corona. Aptum 16, 2/3, S. 134-141.

Marx, Constanze/Weidacher, Georg (2014): Internetlinguistik. Ein Lehr- und Arbeitsbuch. Tübingen: Narr.

Mondada, Lorenza/Svensson, Hanna/Schepen, Nynke van (2015): >Why that not now <: participants' orientations towards several organizational layers in social interaction. In: Bulletin VALS/ASLA 101, S. 51-71.

Niehr, Thomas/Reissen-Kosch, Jana (2018): Volkes Stimme? Zur Sprache des Rechtspopulismus. Berlin: Bibliographisches Institut.

Runkehl, Jens/Schlobinsk, Peter/Siever, Torsten (1998): Sprache und Kommunikation im Internet. In: Muttersprache 2, S. 97-109.

Schatzki, Theodore (2002): The site of the social. A philosophical account of the constitution of social life and change. University Park, PA.: The Pennsylvania State University Press. 
Schmid, Helmut (1994): Probabilistic Part-of-Speech Tagging Using Decision Trees. In: Proceedings of International Conference on New Methods in Language Processing, Manchester, UK.

Schmitz, Ulrich (2015): Einführung in die Medienlinguistik. Darmstadt: WBG.

Schulz-Schaeffer, lngo (2010): Praxis, handlungstheoretisch betrachtet. In: Zeitschrift für Soziologie 39, S. 319-336.

Schüttpelz, Erhard (2016): Infrastrukturelle Medien und öffentliche Medien. In: Media in Action 0 (PrePublication), S. 1-21. URL: https://www.mediacoop.uni-siegen.de/wp-content/uploads/2016/06/ schuettpelz_infrastrukturelle_medien.pdf, letzter Zugriff am 23.05.2021.

Schüttpelz, Erhard/Meyer, Christian (2017): Ein Glossar zur Praxistheorie. »Siegener Version« (Frühjahr 2017). In: Navigationen 17 (1), S. 155-163.

Schüttpelz, Erhard/Meyer, Christian (2018): Charles Goodwin's Co-Operative Action: The Idea and the Argument. In: Media in Action 1, S. 171-188. URL: https://www001.zimt.uni-siegen.de/ojs/index. $\mathrm{php} / \mathrm{mia} / \mathrm{article} / \mathrm{view} / 37$, letzter Zugriff am 23.05.2021.

Seibel, Wolfgang (2016): Verwaltung verstehen. Eine theoriegeschichtliche Einführung. Frankfurt: Suhrkamp.

Transformationen des Populären. Einrichtungsantrag des Geplanten Sonderforschungsbereichs 1472. Universität Siegen 2020 .

Vogel, Friedemann (2012): Das LDA-Toolkit. Korpuslinguistisches Analyseinstrument für kontrastive Diskurs- und Imageanalysen in Forschung und Lehre. In: Zeitschrift für Angewandte Linguistik 57 (1), S. 129-165.

Vogel, Friedemann (2017): Linguistische Imageanalyse Chinas. Theoretisch-methodische Grundlagen und exemplarische Analyse. In: Friedemann Vogel/Wenjian Jia (Hg.): Chinesisch-Deutscher Imagereport. Das Bild Chinas im deutschsprachigen Raum aus kultur-, medien- und sprachwissenschaftlicher Perspektive (2000-2013). Berlin/Boston: De Gruyter Mouton (Sprache und Wissen, 28), S. 48-69.

Weber, Beate (2017): Vorwort zur ersten Auflage, in: Sylvia Löhken/Norbert Brugger (Hg.): Kommunale Redepraxis. 2., erweiterte und aktualisierte Auflage. Stuttgart: Kohlhammer Deutscher Gemeindeverlag, S. VII-VIII.

Werber, Niels (2016): Das Populäre und das Publikum. Inklusion und Attachment. In: Stephan Habscheid/ Christine Hrncal/Raphaela Knipp/Erika Linz (Hg.): Alltagspraktiken des Publikums. Thementeil der Zeitschrift für Literaturwissenschaft und Linguistik 46, S. 469-477. 